\title{
A NOTE ON COMPLETELY POSITIVE GRAPHS (II)
}

\author{
XU CHANGQIN
}

Abstract. A necessary and sufficient condition is given for a doubly nonnegative matrix realization of a cycle to be completely positive. Also some special non-CP graphs are investigated.

\section{Introduction}

Pioneered by M. Hall Jr. in 1958 [2] and investigated by A. Berman [3, 4, 5] and J. H. Drew, C. R. Johnson and Loewy [7] etc., completely positive matrices have been shown their importance not only in the study of block designs in combinatorial analysis [1], but also in establishing economic models [8].

Recall that an $n \times n$ matrix $A$ is said to be completely positive, denoted by $A \in C P_{n}$, if there exist $m$ (entrywise) nonnegative column vectors $b_{1}, \ldots, b_{m}$ such that

$$
A=b_{1} b_{1}^{\prime}+\cdots+b_{m} b_{m}^{\prime}
$$

where' denotes transpose. The smallest such number $m$ is called the factorization index of $A$ and is denoted by $\phi(A)$. An $n \times n$ nonnegative matrix $A$ is called doubly nonnegative, denoted by $A \in D P_{n}$, if it is positive semidefinite. It is known that $D P_{n}=C P_{n}$ for $n \leq 4$; but for $n>4, C P_{n}$ is a proper subset of $D P_{n}$ (see $[1,3]$ ).

Given an $n \times n$ real matrix $A$, let $A(l)$ denote the submatrix of $A$ obtained by deleting the $l$ th row and column of $A$. Let $E_{r s}$ be the square matrix $\left(e_{i j}\right)$ given by

$$
e_{i j}= \begin{cases}1, & \text { if }(i, j)=(r, s) \\ 0, & \text { otherwise. }\end{cases}
$$

For a real symmetric matrix $A$, the graph $G(A)=(V, E)$ of $A$ is defined by: $V=$ $\{1, \ldots, n\}$ and

$$
E=\left\{\{i, j\}: i \neq j, a_{i j} \neq 0, i, j=1, \ldots, n\right\} .
$$

For any vertex $l \in V$, let $N(l)$ denote the set of all neighbours of $l$ in $G$, i.e.

$$
N(l)=\{i:\{i, l\} \in E, i \neq l\} .
$$

Received June 9, 1999; revised September 9, 1999.

2000 Mathematics Subject Classification. 05C50, 05C75, 15A48.

Key words and phrases. Doubly nonnegative matrix, completely positive graph, cycle, factorization index.

Supported by Anhui educational Committee (No: LJ990007). 
By a doubly nonnegative realization of a graph $G$, we mean a matrix $A \in D P_{n}$ for which $G(A)=G$. The set of all such matrices $A$ is denoted by $\Lambda_{G}$. $G$ is called completely positive (abbrev. $C P$ ) if $A \in C P_{n}$ for any $A \in \Lambda_{G}$. It is shown in [3, 4, 5] that a graph $G$ is $C P$ if and only if $G$ does not contain an odd cycle of length greater than 4 . The following known results will be needed in this paper.

Lemma 1 (Theorem 4.3. of [3]). Let $A$ be an $n \times n$ nonzero doubly nonnegative matrix whose graph is acyclic (i.e., without a cycle). Then $A$ is completely positive, and $\phi(A)=\operatorname{rank}(A)=n-m$, where $m$ is the number of singular components of $A$.

Lemma 2 (Theorem 3.5 of [3]). Let $A=\left(a_{i j}\right)$ be an $n \times n$ doubly nonnegative matrix whose graph is $G$. If $G$ has a vertex $l$ which does not lie on a triangle. Then $A$ is completely positive if and only if there exist positive numbers $d_{j}, j \in N(l)$, such that $a_{l l} \geq \sum_{j \in N(l)} \frac{a_{l j}^{2}}{d_{j}}$ and the matrix

$$
H=A(l)-\sum_{j \in N(l)} d_{j} E_{j j}
$$

is completely positive, where the rows and columns of $E_{j j}$ are indexed by $\{1, \ldots, n\} \backslash\{l\}$.

In section 2, we present a necessary and sufficient condition for $A \in \Lambda_{G}$ to be completely positive when $G$ is an odd cycle of length greater than 4 . We also prove that $\phi(A)=n$ for $A \in C P_{n} \bigcap \lambda_{G}$.

In section 3 , we give the definition of nearly vertex(edge) $C P$ graphs and present the structure characterizations of this type of graphs.

\section{A Necessary and Sufficient Condition}

Let $G$ be $C_{2 k+1}$, i.e., a cycle with length $2 k+1$ where $k \geq 2$. We know from [3] that not every matrix in $\Lambda_{G}$ is completely positive and we would like to determine which matrices are. It is obvious that when $A \in \Lambda_{G}, A$ is permutationally similar to a matrix of the form

$$
\left[\begin{array}{cccccc}
a_{11} & a_{12} & 0 & \cdots & 0 & a_{1 n} \\
a_{21} & a_{22} & a_{23} & \cdots & 0 & 0 \\
\vdots & \vdots & \ddots & \ddots & \vdots & \vdots \\
0 & 0 & 0 & \cdots & a_{n-1, n-1} & a_{n-1, n} \\
a_{n 1} & 0 & 0 & \cdots & a_{n, n-1} & a_{n n}
\end{array}\right]
$$

where $a_{i j}=a_{j i}$ for $1 \leq i, j \leq n$. Henceforth, we may assume that $A$ is of this form.

Theorem 1. Let $A \in D P_{n}$ be of the form (1) where $a_{i j} \neq 0$ if and only if $j \equiv$ $i-1, i, i+1(\bmod n)$. Then $A$ is in $C P_{n}$ if and only if there exist two positive numbers $a, b$ such that

(i) $a b=a_{1 n}$. 
(ii) $H=A-\left(a^{2} E_{11}+b^{2} E_{n n}+a_{1 n} E_{1 n}+a_{n 1} E_{n 1}\right) \in D P_{n}$.

Proof. Suppose that there exist $a, b>0$ satisfying (i) and (ii). Let

$$
F=a^{2} E_{11}+b^{2} E_{n n}+a_{1 n} E_{1 n}+a_{n 1} E_{n 1} .
$$

Then $F=\beta \beta^{\prime}$ where $\beta=(a, 0, \ldots, 0, b)^{\prime}$ is a nonnegative column vector of dimension $n$. Since $H \in D P_{n}$ and the graph of $H$ is acyclic, we have $H \in C P_{n}$ by Lemma 1 . So $H=B_{1} B_{1}^{\prime}$ for some $n \times m$ nonnegative matrix $B_{1}$ where $m=\phi(H)$. Put $B=\left[B_{1}, \beta\right]$. Then $B B^{\prime}=B_{1} B_{1}^{\prime}+\beta \beta^{\prime}=H+F=A$. Thus $A$ is completely positive.

Conversely, let $A=\left(a_{i j}\right) \in C P_{n}$ be of the form (1). Then there exists an $n \times m$ nonnegative matrix $B$ such that $A=B B^{\prime}$. Let $B=\left(b_{i j}\right), \beta_{i}=\left(b_{i 1}, \ldots, b_{i m}\right)^{\prime},\left(b_{i j} \geq 0\right)$, and $S_{i}=\left\{k: b_{i k}>0\right\}$. Then

$$
a_{i j}=\left(\beta_{i}, \beta_{j}\right), \quad i, j \in\{1, \ldots, n\}
$$

where we use $\left(\beta_{i}, \beta_{j}\right)$ to denote the usual inner product of $R^{m}$. From (2) and the form of $A$, we have

$$
\left(\beta_{i}, \beta_{j}\right)=0 \text { if and only if } j \not \equiv i-1, i, i+1(\bmod n) .
$$

Then by (3),

$$
S_{i} \bigcap S_{j} \neq \phi \text { if and only if } j \equiv i-1, i, i+1(\bmod n)
$$

Permuting the columns of $B$, if necessary, we may assume that $S_{1} \bigcap S_{n}=\{1,2, \ldots, p\}$, where $1 \leq p<m$. Now we put

$$
a=\left(\sum_{j=1}^{p} b_{1 j}^{2}\right)^{\frac{1}{2}}, \quad b_{1}=\left(\sum_{j=1}^{p} b_{n j}^{2}\right)^{\frac{1}{2}} .
$$

Since

$$
a b_{1}=\left[\left(\sum_{j=1}^{p} b_{1 j}^{2}\right)\left(\sum_{j=1}^{p} b_{n j}^{2}\right)\right]^{\frac{1}{2}} \geq \sum_{j=1}^{p} b_{1 j} b_{n j}=\left(\beta_{1}, \beta_{n}\right)=a_{1 n}>0,
$$

there exists a real number $b, 0<b \leq b_{1}$ such that $a b=a_{1 n}$. Next we set $y=\sqrt{b_{1}^{2}-b^{2}}$, and define $\tilde{\beta}_{i} \in R^{(m-p+2)}, i=1, \ldots, m$ by

$$
\begin{aligned}
\tilde{\beta}_{1}= & \left(0, a, b_{1, p+1}, b_{1, p+2}, \ldots, b_{1 m}\right)^{\prime} \\
\tilde{\beta}_{2}= & \left(0,0, b_{2, p+1}, \ldots, b_{2 m}\right)^{\prime} \\
& \vdots \\
\tilde{\beta}_{n-1}= & \left(0,0, b_{n-1, p+1}, \ldots, b_{n-1, m}\right)^{\prime} \\
\tilde{\beta}_{n}= & \left(y, b, b_{n, p+1}, \ldots, b_{n m}\right)^{\prime}
\end{aligned}
$$

Also let $\tilde{B}=\left[\tilde{\beta}_{1}, \ldots, \tilde{\beta}_{n}\right]^{\prime}$. Then we have $A=\tilde{B} \tilde{B}^{\prime}$, and $H=\hat{B} \hat{B}^{\prime}$, where $\hat{B}$ is obtained from $\tilde{B}$ by replacing the $a$ in $\tilde{\beta}_{1}$ and the $b$ in $\tilde{\beta}_{n}$ both by 0 . This completes the proof of the theorem. 
Corollary 2. Let $A \in C P_{n}$ and suppose that $G(A)$ is an odd cycle of length greater than 4. Then $\phi(A)=n$.

Proof. We may assume that $A$ is of the form (1). First we use the proof of the converse part of Theorem 1 , but with $m=\phi(A)$, to prove $\phi(A) \geq n$ : Condition (4) implies that for each $i=1,2, \ldots, n, S_{i} \bigcap S_{i-1} \neq \phi$ and $S_{i} \bigcap S_{i+1} \neq \phi$ (but $S_{i-1} \bigcap S_{i+1}=\phi$ ), from which inductively it follows that

$$
\left|S_{1} \bigcup S_{2} \bigcup \cdots \bigcup S_{i}\right| \geq i+1
$$

for all $i=1, \ldots, n-1$, where we use $|S|$ to denote the cardinality of a set $S$; hence we have

$$
\phi(A)=m \geq\left|S_{1} \bigcup S_{2} \bigcup \cdots \bigcup S_{n}\right| \geq n .
$$

It suffices to prove $\phi(A) \leq n$. Without loss of generality, we may assume that $a_{11}=\cdots=a_{n n}=1$. Moreover, denote $a_{i, i+1}$ by $a_{i}, i=1, \ldots, n$, where $a_{n, n+1}=a_{n 1}$. Since $A \in C P_{n}$, there exist $a, b>0$ such that both of the following hold:

(I) $1 \geq \frac{a_{n}^{2}}{a}+\frac{a_{n-1}^{2}}{b}$;

(II) $H_{1}=A(n)-\left(a E_{11}+b E_{n-1, n-1}\right) \in D P_{n-1}$.

If the inequality in (I) is strict, we decrease gradually the value of $b$ while keeping the value of $a$ until the equality occurs in (I). (Note that in doing so, condition (II) still holds.) If the remaining matrix $H_{1}$ is positive definite, we increase gradually the value of $a$ while decreasing the value of $b$ to keep the equality in (I) until the matrix $H_{1}$ becomes singular. (Note that the process must stop for some $0<a \leq 1$ since $a_{i i}=1$ by assumption.) So we may assume that the equality holds in (I), and also that the matrix $H_{1}$ is singular. By Lemma $1, \phi\left(H_{1}\right)=n-2$. Let $H_{1}=B_{2} B_{2}^{\prime}$ be a factorization of $H_{1}$ where $B_{2}$ is an $(n-1) \times(n-2)$ nonnegative matrix. Set

$$
B_{1}=\left[\sqrt{a} e_{1}, B_{2}, \sqrt{b} e_{n-1}\right]
$$

where $e_{i}$ is the $i^{t h}$ coordinate vector of $R^{n-1}$. Then $B_{1}$ is a $(n-1) \times n$ nonnegative matrix. Putting

$$
x=\left(\frac{a_{n}}{\sqrt{a}}, 0, \ldots, 0, \frac{a_{n-1}}{\sqrt{b}}\right)
$$

where $x \in R_{+}^{n}$, and writing

$$
B=\left[\begin{array}{c}
B_{1} \\
x
\end{array}\right]
$$

we have $A=B B^{\prime}$, from which we can see easily that $\phi(A) \leq n$. The proof is completed.

The following interesting alternative argument for $\phi(A) \leq n$ is based on results in [3] (which is simpler than the above). Since $A \in C P_{n}$, by Theorem 3.5 of [3] (with $l=1$ ), there exist positive numbers $d_{2}, d_{n}$ such that both of the following hold:

(a) $1>\frac{a_{12}^{2}}{d_{2}}+\frac{a_{1 n}^{2}}{d_{n}}$; 
(b) $H=A(1)-\left(d_{2} E_{22}+d_{n} E_{n, n}\right) \in C P_{n-1}$.

If the matrix $H$ is not singular, we increase the value of $d_{2}$ gradually (but keeping the value of $d_{n}$ ) until the matrix $H$ becomes singular. Note that in doing so the inequality (a) remains valid. So we may assume that $H$ is singular. Then by Theorem 3.1 of [3] we have $\phi(A) \leq \phi(H)+2 \leq(n-2)+2=n$ where $\phi(H) \leq n-2$ follows from the fact that $G(H)$ is acyclic (and Lemma 1 ).

\section{Nearly CP Graphs}

A graph $G$ is called nearly vertex (resp. edge) $C P$ if $G$ is not $C P$ but $G-\{v\}$ (resp. $G-e$ ) is $C P$ for any $v \in V(G)$ (resp. $e \in E(G)$ ).

The following facts are obvious.

Proposition 1. Every nearly vertex $C P$ graph is connected and contains an odd cycle of length greater than 4 .

Proposition 2. Every connected nearly edge $C P$ graph is nearly vertex $C P$.

Proposition 3. A graph of order 5 is nearly vertex $C P$ if and only if it contains a cycle of length 5 .

For graphs of order greater than 5 , we have the following result.

Theorem 3. Let $G=(V, E)$ be a graph with $n$ vertices where $n>5$. Then $G$ is nearly vertex $C P$ if and only if it is one of the following graphs:

1. $C_{2 k+1}$ where $k \geq 3$ is an integer;

2. $C_{2 k+1}+e$ where $k \geq 3$ and $e$ is an edge and lies in a triangle

3. $C_{2 k+1}+e_{1}+e_{2}$ where $e_{1}=\left\{x_{1}, x_{3}\right\}, e_{2}=\left\{x_{2}, x_{4}\right\}$, and $\left\{x_{1}, x_{2}\right\},\left\{x_{2}, x_{3}\right\},\left\{x_{3}, x_{4}\right\}$ are edges of $C_{2 k+1}$.

Proof. The "if" part can be verified readily. Now we consider the "only if" part. Since $G$ is not $C P, G$ contains an odd cycle $C_{2 k+1}$ where $k \geq 2$. If $V\left(C_{2 k+1}\right) \neq V$, we choose $v \in V \backslash V\left(C_{2 k+1}\right)$. Then $G-\{v\}$ still contains the cycle $C_{2 k+1}$, and so $G-\{v\}$ is not $C P$, a contradiction. Hence $V=V\left(C_{2 k+1}\right)$.

Since $n=2 k+1>5$, we have $k \geq 3$. Suppose $G$ is not the cycle $C_{2 k+1}$. Choose an edge $e \in E \backslash E\left(C_{2 k+1}\right)$. Then the edge $e$, together with one part of $C_{2 k+1}$, forms an odd cycle, say $C_{l}$, where $l \geq 3$ is odd, and $e$, with the one part of $C_{2 k+1}$, forms an even cycle, say $C_{2 r}$ where $2 r=2 k+1-l+2=2 k-1+3$. Choose a vertex $v \in V\left(C_{2 r}\right) \backslash V\left(C_{l}\right)$. Since $G$ is nearly vertex $C P, G-\{v\}$ is $C P$. But $G-\{v\}$ contains the odd cycle $C_{l}$, so we must have $l=3$. In other words, $e$ lies in a triangle. If $G$ is an odd cycle plus an edge, then $G=G_{2 k+1}+e_{1}$, and $G$ is of type (2). Suppose $G$ is an odd cycle plus more than one edge. Denote the above edge $e$ by $e_{1}$ and let $T_{1}=\{u, v, w\}$ be the triangle containing $e_{1}$ with $e_{1}=\{u, v\}$. Choose an edge $e_{2}$ that lies outside $C_{2 k+1}+e_{1}$. From the preceding proof, it is clear that $e_{2}$ also lies on a triangle $T_{2}$ for which two of its edges 
belong to the cycle $C_{2 k+1}$, say $T_{2}=\{x, y, z\}$ with $e_{2}=\{x, z\}$. If $T_{2}$ has no common edges with $T_{1}$ (but $T_{2}$ may have a common vertex with $T_{1}$ ), then the graph $G-\{w\}$ contains the cycle $C_{2 k+1}-\{w, y\}$ as its subgraph. But the length of the latter cycle is $2 k+1-4+2=2(k-1)+1 \geq 5$. Hence $G-\{w\}$ is not $C P$, which contradicts the definition of $G$. Thus $T_{1}$ and $T_{2}$ must have an edge in common. By a similar argument, we can see that $G$ cannot have any other edges. Therefore, $G$ is type (3). The proof is completed.

We end our discussion by treating nearly edge $C P$ graphs.

Corollary 4. A connected graph $G$ is nearly edge $C P$ if and only if $G$ is an odd cycle with length greater than 4 .

Proof. The sufficiency of the condition is obvious.

Since $G$ is not $C P, G$ contains an odd cycle $C_{2 k+1}$, where $k \geq 2$. If $G$ has an edge $e$ that does not lie on the cycle $C_{2 k+1}$, then clearly $G-e$ is not $C P$, a contradiction. So all edges of $G$ lie on $C_{2 k+1}$. Furthermore, all vertices of $G$ belong to $C_{2 k+1}$ as $G$ is connected. So we have $G=C_{2 k+1}$.

\section{Acknowledgements}

I wish to express my gratitude to Professor Abraham Berman for his inspiring series of lectures during his visit to The University of Science and Technology of China in September, 1996. I also thank the anonymous referee for his patient corrections which significantly improved the paper and for his presentation of the alternative argument for the inequality $\phi(A) \leq n$ in the proof of Corollary 2 .

\section{References}

[1] M. Hall Jr., Combinatorial Theory (2nd ed.), Wiley, New York, 1986.

[2] M. Hall Jr., Surveys in Applied Mathematics, Wiley, New York, 4(1958), 35-104.

[3] A. Berman and D. Hershkowitz, Combinatorial results on completely positive matrices, Linear Algebra Apple., 95(1987), 111-125.

[4] A. Berman and R. Grone, Bipartite completely positive graphs, Proc. Cambridege Philos. Soc., 103(1988), 269-276.

[5] A. Berman and N. Kogan, Characterization of completely positive graphs, Discrete Math., 114(1993), 297-304.

[6] A. Berman and R. Plemmons, Nonnegative Matrices in the Mathematical Sciences, Academic Press, New York, 1979.

[7] J. H. Drew, C. R.Johnson and R. Loewy, Completely positive matrices associated with Mmatrices, Linear and Multilinear Algebra, 37(1994), 303-310.

[8] T. L. Markham, Factorizations of completely positive matrices, Proc. Cambirdge Philos. Soc., 69(1971), 53-58.

[9] L. J. Gray and D. G. Wilson, Nonnegative factorization of positive semidefinite nonnegative matrices, Linear Algebra Appl., 31(1980), 119-127.

Department of Mathematics, Anhui University, Hefei, Anhui 230039 P. R. China.

E-mail: cqxu@mars.ahu.edu.cn 